$\mathrm{SPhT} / 92-053$

\title{
Partition Functions, Intertwiners and the Coxeter Element
}

\author{
Patrick Dorey $^{\dagger}$ \\ Service de Physique Théorique de Saclayl, \\ 91191 Gif-sur-Yvette cedex, France
}

\begin{abstract}
The partition functions of Pasquier models on the cylinder, and the associated intertwiners, are considered. It is shown that earlier results due to Saleur and Bauer can be rephrased in a geometrical way, reminiscent of formulae found in certain purely elastic scattering theories. This establishes the positivity of these intertwiners in a general way and elucidates connections between these objects and the finite subgroups of $S U(2)$. It also offers the hope that analogous geometrical structures might lie behind the so-far mysterious results found by Di Francesco and Zuber in their search for generalisations of these models.
\end{abstract}

May 1992

$\dagger$ dorey@poseidon.saclay.cea.fr

1 Laboratoire de la Direction des Sciences de la Matière du Commissariat à l'Energie Atomique 


\section{Introduction}

Consider a critical lattice model defined, not on a torus, but on a cylinder of circumference $l$ and length $l^{\prime}$. Once boundary conditions $A$ and $B$ have been specified at the two ends, a partition function $Z_{A B}$ can be defined which, for large enough lattices, will be a function of the ratio $l / l^{\prime}$ alone. The continuum limit being a conformal field theory, this function should be expressible in terms of characters of a (perhaps extended) Virasoro algebra - just one copy in this case, since the surface has a boundary. Thus an expression of the form

$$
Z_{A B}\left(l, l^{\prime}\right) \sim \sum_{r} n_{A B}^{r} \chi_{r}(q), \quad q=e^{-\pi l / l^{\prime}}
$$

is expected [1], where $r$ runs over the possible algebra representations appearing in the spectrum of $H_{A B}$, the Hamiltonian propagating states (on a line length $l^{\prime}$, with boundary conditions $A$ and $B$ ) around the cylinder, and $\chi_{r}(q)$ is the corresponding character. The numbers $n_{A B}^{r}$ are thus multiplicities, and as such should be non-negative integers. In theories where the $\chi_{r}$ 's are the characters of a (maximally-extended) chiral algebra, in terms of which the torus partition function is diagonal, Cardy [2] subsequently indicated a correspondence between the coefficients $n_{A B}^{r}$ and the fusion rules à la Verlinde [3] of this algebra.

In certain specific cases for the Pasquier models - generalised RSOS models where the heights live on the Dynkin diagram of some simply-laced Lie algebra $G$ [ [ - equation (1.1) was confirmed by explicit calculation in ref. [5]. Among other things, the case where the heights at the two ends are constrained to be equal to $a$ and $b$ respectively was examined ( $a$ and $b$ labelling two nodes on the Dynkin diagram of $G$ ), with the result

$$
Z_{a b}^{(G)}\left(l, l^{\prime}\right) \sim \sum_{\lambda=1}^{h-1} V_{a b}^{\lambda} \chi_{1, \lambda}(q)
$$

where

$$
V_{a b}^{\lambda}=\sum_{s \in\left\{\begin{array}{c}
\text { exponents }\} \\
\text { of } G
\end{array}\right.} \frac{\phi_{\lambda}^{(s)}}{\phi_{1}^{(s)}} q_{a}^{(s)} q_{b}^{(s)} .
$$

In the first equation, $h$ is the Coxeter number of $G$ and $\chi_{1, \lambda}$ is a Virasoro character from the first row of the Kac table for the central charge $c=1-6 / h(h-1)$ of the model, while in the second $\phi^{(s)}$ and $q^{(s)}$ are eigenvectors of the $A_{h-1}$ and $G$ Cartan matrices respectively, both with eigenvalue $2-2 \cos \pi s / h$. Explicitly, $\phi_{\lambda}^{(s)}=\sqrt{ }(2 / h) \sin (\pi s \lambda / h)$. If an exponent $s$ of $G$ 
occurs more than once, there is a freedom to rotate among the corresponding eigenvectors $q^{(s)}$, but this causes no ambiguity since their total contribution to (1.3) is a quadratic form.

The result (1.2) actually holds even before the continuum limit is taken, on replacing each $\chi_{1, \lambda}(q)$ on the right hand side by $Z_{1 \lambda}^{\left(A_{h-1}\right)}\left(l, l^{\prime}\right)$, the partition function for an $A_{h-1}$ model with boundary spins 1 and $\lambda$, evaluated in the same geometry as the left hand side [6, [7. (Consistency with (1.2) follows from the fact that $Z_{1 \lambda}^{\left(A_{h-1}\right)}\left(l, l^{\prime}\right) \sim \chi_{1, \lambda}(q)$ in the continuum limit [5].) This result justifies the use of the term 'intertwiner' for the matrix $V^{\lambda}$, and connects with the more algebraic notions that for finite systems the Virasoro algebra should be replaced by that of Temperley and Lieb [8]. Thus for a lattice of width $l^{\prime}$ with boundary conditions $a$ and $b$, the space of states is spanned by the set $\mathcal{P}_{a b}^{\left(l^{\prime}\right) G}$ of paths of length $l^{\prime}$ running from $a$ to $b$ on the Dynkin diagram of $G$, and this space supports a representation $\mathcal{R}_{a b}^{\left(l^{\prime}\right) G}$ of the Temperley-Lieb algebra. The (modified) trace for this representation decomposes as

$$
\operatorname{tr}_{\mathcal{R}_{a b}^{\left(l^{\prime}\right) G}}(.)=\sum_{\lambda} V_{a b}^{\lambda} \operatorname{tr}_{\mathcal{R}_{1 \lambda}^{\left(l^{\prime}\right) A_{h-1}}}(.)
$$

into traces over $A$-type representations $\mathcal{R}_{1 \lambda}^{\left(l^{\prime}\right) A_{h-1}}$, a formula which contains the abovementioned result for finite-geometry partition functions as a special case日. The $V^{\lambda}$ 's are again multiplicities, but this time of representations of Temperley-Lieb rather than of Virasoro.

In fact, the authors of [6, [] were primarily interested in these questions as a warm-up exercise in their search for generalisations of the Pasquier models. This also led them study the numbers $V_{a b}^{\lambda}$ on a more axiomatic basis, thinking of them as (non-negative integer valued) matrix representations of a Verlinde algebra. The $V^{\lambda}$ defined above exhaust the possibilities for the fusion algebras of the affine $\widehat{S U(2)})_{k}$ Kac-Moody theories at level $k=h-2$. For further information on all this material, see the review articles 911 .

Despite the many results that were uncovered in the course of these investigations, it is likely that there remain some underlying principles yet to be discovered. To give a small example, the fact that the the $V_{a b}^{\lambda}$ are non-negative, clear from their interpretation

2 Strictly speaking, (1.4) is not always a decomposition: for $G=D_{n}$, and $a$ and $b$ two extremal nodes with only one of them a spinor, there is only one non-zero term on the right hand side of (1.4), at $\lambda=n-1$. 
as multiplicities, is not at all evident from (1.3) or its generalisations, and was only established case-by-case (even their integrality is not immediately obvious). For this reason it seems worthwhile to understand the $\widehat{S U(2)})_{k}$ examples, which are at least completely classified, as thoroughly as possible. The purpose of this paper is to report an observation which may help towards this goal, allowing (1.3) to be rewritten in a geometrical way for which integrality and non-negativity are manifest, and for which various other apparent coincidences (such as a connection with the finite subgroups of $S U(2)$ ) become rather less mysterious. The necessary geometrical information is established in section two, while following sections relate this to (1.3) and discuss various implications.

\section{Geometrical details}

To set up notations, this section starts with a brief review of some relevant information about the Coxeter elements of the Weyl group of a simply-laced Lie algebra $G$. For further explanations and applications, see [12 22]. This material will then be used to derive expressions for certain inner products between roots and/or weights, some of which turn out to reproduce (1.3). The restriction to the simply-laced cases avoids the need to

distinguish left and right eigenvectors of the Cartan matrix $C_{a b}^{(G)}$, and will be sufficient for current needs.

Splitting the simple roots of $G$ into two internally orthogonal sets, $\Delta=\left\{\alpha_{\bullet}\right\} \cup\left\{\alpha_{\circ}\right\}$, according to a two-colouring of the Dynkin diagram of $G, \sum_{\bullet^{\prime}}, \sum_{\circ^{\prime}}, \sum_{s}$ will denote summation over the black indices, the white indices and the exponents of $G$, respectively. The eigenvectors $q^{(s)}$ of $C_{a b}^{(G)}$ satisfy

$$
C^{(G)} q^{(s)}=\left(2-2 \cos \theta_{s}\right) q^{(s)}
$$

where $\theta_{s}=\pi s / h$ and $s$ is one of the exponents. These eigenvectors will be normalised to length one, and the residual phases picked so as to satisfy

$$
q_{\bullet}^{(s)}=q_{\bullet}^{(h-s)} \quad q_{\circ}^{(s)}=-q_{\circ}^{(h-s)} .
$$

If $s=h / 2$ is an exponent, then this choice in fact fixes which roots are black and which white - for all indices in the white set, $q_{\circ}^{(h / 2)}$ vanishes. While the choice (2.2) serves to fix various quantities which arise during the working, the final results will not depend on it. 
Letting $r_{i}$ denote the Weyl reflection for the simple root $\alpha_{i}$, set

$$
w_{\{\bullet\}}=\prod_{\bullet} r_{\bullet} \quad w_{\{0\}}=\prod_{0^{\prime}} r_{\circ^{\prime}}
$$

so that $w=w_{\{\bullet\}} w_{\{0\}}$, the product of two involutions, is a Coxeter element. (The utility of this particular choice of Coxeter element was pointed out by Steinberg [12].) An invariant subspace for $w_{\{\bullet\}}, w_{\{\circ\}}$ and hence for $w$ is spanned by

$$
a_{\{\bullet\}}^{(s)}=\sum_{\bullet} q_{\bullet^{\prime}}^{(s)} \alpha_{\bullet} \quad a_{\{\circ\}}^{(s)}=\sum_{\circ^{\prime}} q_{\circ^{\prime}}^{(s)} \alpha_{\circ^{\prime}}
$$

The invariance stems from the facts, not too hard to verify, that $w_{\{\bullet\}}$ and $w_{\{0\}}$ act on these vectors in the following way:

$$
\begin{array}{ll}
w_{\{\bullet\}} a_{\{\bullet\}}^{(s)}=-a_{\{\bullet\}}^{(s)} & w_{\{\bullet\}} a_{\{\circ\}}^{(s)}=a_{\{\circ\}}^{(s)}+2 \cos \theta_{s} a_{\{\bullet\}}^{(s)} \\
w_{\{\circ\}} a_{\{\circ\}}^{(s)}=-a_{\{\circ\}}^{(s)} & w_{\{\circ\}} a_{\{\bullet\}}^{(s)}=a_{\{\bullet\}}^{(s)}+2 \cos \theta_{s} a_{\{\circ\}}^{(s)}
\end{array}
$$

Following from these relations, $\left|a_{\{\bullet\}}^{(s)}\right|=\left|a_{\{\circ\}}^{(s)}\right|=1$ (except if $h / 2$ is an exponent, in which case $\left|a_{\{\bullet\}}^{(h / 2)}\right|=\sqrt{ } 2$ and $\left.a_{\{\circ\}}^{(h / 2)}=0\right)$, and $\left(a_{\{\bullet\}}^{(s)}, a_{\{\circ\}}^{(s)}\right)=-\cos \theta_{s}$. Dual to (2.4), the simple roots can be expanded in terms of the invariant subspaces:

$$
\alpha_{\bullet}=\sum_{s} q_{\bullet}^{(s)} a_{\{\bullet\}}^{(s)} \quad \alpha_{\circ}=\sum_{s} q_{\circ}^{(s)} a_{\{\circ\}}^{(s)}
$$

The duality referred to here is between the set of points on the Dynkin diagram and the set of exponents; there is of course also the vector space duality between the simple roots $\alpha_{a}$ and the fundamental weights $\lambda_{b}$, defined through $\left(\alpha_{a}, \lambda_{b}\right)=\delta_{a b}$. The invariant subspaces for $w$ are also spanned by objects constructed from these weights, namely

$$
l_{\{\bullet\}}^{(s)}=\sum_{\bullet} q_{\bullet^{\prime}}^{(s)} \lambda_{\bullet} \quad l_{\{\circ\}}^{(s)}=\sum_{\circ^{\prime}} q_{\circ^{\prime}}^{(s)} \lambda_{\circ^{\prime}},
$$

in terms of which the fundamental weights themselves expand as

$$
\lambda_{\bullet}=\sum_{s} q_{\bullet}^{(s)} l_{\{\bullet\}}^{(s)} \quad \lambda_{\circ}=\sum_{s} q_{\circ}^{(s)} l_{\{\circ\}}^{(s)} .
$$

To check the various properties, note that $\left(l_{\{\bullet\}}^{(s)}, a_{\{\bullet\}}^{(s)}\right)=\left(l_{\{\circ\}}^{(s)}, a_{\{\circ\}}^{(s)}\right)=1 / 2$ (or 1,0 respectively if $s=h / 2$ ), and that as a result of (2.2),

$$
\begin{array}{cc}
a_{\{\bullet\}}^{(s)}=a_{\{\bullet\}}^{(h-s)} & a_{\{\circ\}}^{(s)}=-a_{\{\circ\}}^{(h-s)} \\
l_{\{\bullet\}}^{(s)}=l_{\{\bullet\}}^{(h-s)} & l_{\{\circ\}}^{(s)}=-l_{\{\circ\}}^{(h-s)}
\end{array}
$$


In addition, $\left|l_{\{\bullet\}}^{(s)}\right|=\left|l_{\{\circ\}}^{(s)}\right|=1 /\left(2 \sin \theta_{s}\right)$ (or $1 / \sqrt{ } 2$ and 0 if $\left.s=h / 2\right)$, and the angle between $l_{\{\bullet\}}^{(s)}$ and $l_{\{\circ\}}^{(s)}$ is $\theta_{s}$. Rather than write down yet more identities, the data is summarised in figure 1. Noting from (2.5) that, in the subspace depicted, $w_{\{\bullet\}}$ acts as the reflection leaving $l_{\{\circ\}}^{(s)}$ fixed and vice versa, it is then clear that $w$ itself is a rotation (in the direction from $l_{\{\bullet\}}$ to $\left.l_{\{\circ\}}\right)$ of $2 \theta_{s}=2 \pi s / h$.

To see this action explicitly, a mixed basis is perhaps the most convenient, such as that provided by $l_{\{\bullet\}}^{(s)}$ and $a_{\{\circ\}}^{(s)}$. Examining figure 1, it can be seen that 2.8 generalises to

$$
w^{p} \lambda_{a}=\sum_{s} q_{a}^{(s)}\left(\cos \left(2 p+u_{a}\right) \theta_{s} l_{\{\bullet\}}^{(s)}+\frac{\sin \left(2 p+u_{a}\right) \theta_{s}}{2 \sin \theta_{s}} a_{\{\circ\}}^{(s)}\right),
$$

a formula which holds good even if $h / 2$ is among the exponents. Both black and white indices have been accounted for here, via the convention that $u_{a}=0$ if $a \in\{\bullet\}, u_{a}=1$ if $a \in\{0\}$.

Enough information has now been gathered to write down expressions for various inner products. First, consider $\left(w^{p} \lambda_{a}, \lambda_{b}\right)$. From (2.10),

$$
\begin{array}{r}
\left(w^{p} \lambda_{a}, \lambda_{b}\right)=\sum_{s, s^{\prime}} q_{a}^{(s)}\left(\cos \left(2 p+u_{a}\right) \theta_{s} l_{\{\bullet\}}^{(s)}+\frac{\sin \left(2 p+u_{a}\right) \theta_{s}}{2 \sin \theta_{s}} a_{\{\circ\}}^{(s)}\right) \\
\times q_{b}^{\left(s^{\prime}\right)}\left(\cos u_{b} \theta_{s^{\prime}} l_{\{\bullet\}}^{\left(s^{\prime}\right)}+\frac{\sin u_{b} \theta_{s^{\prime}}}{2 \sin \theta_{s^{\prime}}} a_{\{\circ\}}^{\left(s^{\prime}\right)}\right) .
\end{array}
$$

Now $\left(l_{\{\bullet\}}^{(s)}, a_{\{0\}}^{\left(s^{\prime}\right)}\right)$ is zero for all $s, s^{\prime}$, as are all other terms if $s^{\prime}$ is not equal to either $s$ or $h-s$. Noting for the latter case that, no matter what the colour of the index $b, q_{b}^{(s)} \cos u_{b} \theta_{s}$ is unchanged under $s \rightarrow h-s$ while $q_{b}^{(s)} \sin u_{b} \theta_{s}$ is negated, the identities given above together with a little algebra reduce (2.11) to

$$
\left(w^{p} \lambda_{a}, \lambda_{b}\right)=\sum_{s} q_{a}^{(s)} q_{b}^{(s)} \frac{\cos \left(2 p+u_{a b}\right) \theta_{s}}{2 \sin ^{2} \theta_{s}},
$$

where $u_{a b}=u_{a}-u_{b}$. This expression is almost (1.3), but not quite. For this, the inner products between roots and weights are needed. Within the set $\Phi$ of all the roots, a complete set of orbit representatives for $w$ is provided by the elements $\phi_{a}=\left(1-w^{-1}\right) \lambda_{a}$ [13]. For the Coxeter element in use here, their relationship with the simple roots is

$$
\phi_{\bullet}=w_{\{\circ\}} \alpha_{\bullet} \quad \phi_{\circ}=\alpha_{\circ}
$$


A summary of some of the useful properties of these roots in the context of purely elastic S-matrices can be found in [17], and here too they turn out to be a convenient choice. The inner product of $\lambda_{a}$ with $w^{-p} \phi_{b}$ follows very quickly from (2.12), since

$$
\begin{aligned}
\left(\lambda_{a}, w^{-p} \phi_{b}\right) & =\left(\lambda_{a}, w^{-p}\left(1-w^{-1}\right) \lambda_{b}\right) \\
& =\left(w^{p} \lambda_{a}, \lambda_{b}\right)-\left(w^{p+1} \lambda_{a}, \lambda_{b}\right) .
\end{aligned}
$$

After a small amount of work,

$$
\left(\lambda_{a}, w^{-p} \phi_{b}\right)=\sum_{s} q_{a}^{(s)} q_{b}^{(s)} \frac{\sin \left(2 p+1+u_{a b}\right) \theta_{s}}{\sin \theta_{s}} .
$$

The same procedure repeated one more time gives the final identity of this section, namely

$$
\left(\phi_{a}, w^{-p} \phi_{b}\right)=\sum_{s} 2 q_{a}^{(s)} q_{b}^{(s)} \cos \left(2 p+u_{a b}\right) \theta_{s} .
$$

The case $a=b, p=0$ gives a simple check that there have been no mistakes in the working.

Equations (2.12), (2.14) and (2.15) allow various identities between inner products to spotted, some of which were listed in [16]:17]. Actually, the identities given in [16] involved the simple roots $\alpha_{a}$ rather than the orbit representatives $\phi_{a}$; to obtain formulae for their inner products from those given above, start by substituting for $\phi_{a}$ and/or $\phi_{b}$ using (2.13). Then results such as $w^{-p} w_{\{\circ\}}=w_{\{\circ\}} w^{p}, w_{\{\bullet\}} \lambda_{\circ}=\lambda_{\circ}$ and $w_{\{\circ\}} \alpha_{\circ}=-\alpha_{\circ}$, together with the Weyl-group invariance of the inner product, rapidly convert (2.14) and (2.15) into the desired forms.

\section{Consequences}

Comparing (2.14) with (1.3) and recalling the specific form of the $A_{h-1}$ eigenvectors gives observation advertised in the introduction:

$$
V_{a b}^{2 p+1+u_{a b}}=\left(\lambda_{a}, w^{-p} \phi_{b}\right)
$$

Since in (1.2) the index $\lambda$ ran over all integers from 1 to $h-1$, it might appear that only half of the $V^{\lambda}$ 's have been given a geometrical interpretation by (3.1). However the other $V^{\lambda}$ 's are zero, a fact which can be traced to the existence of a $\mathbf{Z}_{2}$ charge [6], the index colour. To see this it suffices to note that, from (2.2) and the form of $\phi_{\lambda}^{(s)}$,

$$
\frac{\phi_{\lambda}^{(s)}}{\phi_{1}^{(s)}} q_{a}^{(s)} q_{b}^{(s)}=(-1)^{\lambda-1-u_{a b}} \frac{\phi_{\lambda}^{(h-s)}}{\phi_{1}^{(h-s)}} q_{a}^{(h-s)} q_{b}^{(h-s)} .
$$


Hence for the sum in (1.3) to be non-vanishing, $\lambda-1-u_{a b}$ must be even, $2 p$ say, a requirement which exactly reproduces the cases covered by (3.1).

The root systems under discussion being simply laced, the fundamental weights are dual to the simple roots and the integrality of the $V^{\lambda}$ 's is immediate. That they are non-negative is just a little harder, but has in fact already been discussed, albeit in the apparently very different context of exact S-matrices, in [17,22].

Comparing (3.1) with (1.2), what is needed is a proof that the inner products $\left(\lambda_{a}, w^{-p} \phi_{b}\right)$ are non-negative for $p$ in the range $1 \leq 2 p+1+u_{a b} \leq h-1$. This will certainly be true if $w^{-p} \phi_{b} \in \Phi^{+}$for all such $p$, where $\Phi^{+}$is the set of positive roots. This property was relevant for a discussion of certain analyticity properties of the S-matrix formulae given in [16], and essentially algebraic demonstrations were indicated in [17,22]; for the sake of variety the following gives a rather more geometrical approach.

By the Perron-Frobenius theorem (and perhaps after an overall sign change), $q_{a}^{(1)}>0$ for all $a, q^{(1)}$ being the eigenvector of $C^{G}$ with the smallest eigenvalue. Hence the vector

$$
l^{(1)}=l_{\{\bullet\}}^{(1)}+l_{\{\circ\}}^{(1)}=\sum_{a} q_{a}^{(1)} \lambda_{a}
$$

is a strictly positive linear combination of the fundamental weights. As a result, for any $\alpha \in \Phi$,

$$
\alpha \in \Phi^{+} \Longleftrightarrow\left(l^{(1)}, \alpha\right)>0
$$

Since $l^{(1)}$ lies in the $s=1$ eigenspace of $w$, this means that the positivity of a root can be ascertained simply by looking at its $s=1$ projection. Figure 2 illustrates this, showing the projections of the roots in two typical orbits. (As each orbit contains nine roots, this is in fact the $A_{8}$ case.) This gives a visual characterisation of the positive and negative roots in each orbit, and it is now easy to see that if $h$ is even the positive roots are $w^{-p} \phi_{b}$ for $p=0, \ldots, h / 2-1$, while for $h$ odd $\left(i e\right.$ for $A_{2 n}$ ) the relevant range for $p$ is $0, \ldots,(h-3) / 2$ if $b \in\{\bullet\}$, and $0, \ldots,(h-1) / 2$ if $b \in\{\circ\}$. Since these ranges always include those relevant for the $V^{\lambda}$ that appear in (1.2), the non-negativity of these numbers has now been shown to follow from general principles.

Less visually but more explicitly, the formulae in the last section lead to the following expression:

$$
\left(l^{(1)}, w^{-p} \phi_{b}\right)=q_{b}^{(1)} \frac{\sin \left(2 p+\frac{3}{2}-u_{b}\right) \frac{\pi}{h}}{\sin \frac{\pi}{2 h}} .
$$


Positivity is thus equivalent to $0<2 p+\frac{3}{2}-u_{b}<h$, or (adding $u_{a}-1 / 2$ throughout) $2 p+1+u_{a b}$ lying between $u_{a}-1 / 2$ and $h-1 / 2+u_{a}$. Whatever the value of $u_{a}$ (0 or 1 ), this includes the range $1, \ldots, h-1$ needed to establish that, as claimed, the $V^{\lambda}$ 's are non-negative.

To sum up, formula (1.2) for the partition function can now be rewritten as

$$
Z_{a b}^{(G)} \sim \sum_{w^{-p} \phi_{b} \in \Phi^{+}}\left(\lambda_{a}, w^{-p} \phi_{b}\right) \chi_{1,2 p+1+u_{a b}} .
$$

This expression appears to contain extra terms over (1.2), since, while the range for $p$ implied by positivity of $w^{-p} \phi_{b}$ includes that relevant for (1.2), it does not necessarily coincide with it exactly. The possible extra terms involve $\chi_{1,0}$ or $\chi_{1, h}$; however all is well since their coefficients $\left(\lambda_{a}, w^{-p} \phi_{b}\right)$ are forced to be zero by the relation

$$
\left(\lambda_{a}, w^{-p} \phi_{b}\right)=-\left(\lambda_{a}, w^{p+1+u_{a b}} \phi_{b}\right)
$$

and the fact that if $2 p+1+u_{a b}$ is equal to 0 or $h$, we also have $w^{-p} \phi_{b}=w^{p+1+u_{a b}}$.

To give a little more geometrical sense to (3.6), some notation can be borrowed from [17]. For any pair of roots $\alpha, \beta \in \Phi$, define an integer $u(\alpha, \beta)$ modulo $2 h$ by

$$
u(w \alpha, \beta)=u(\alpha, \beta)+2, \quad u(\alpha, \beta)=-u(\beta, \alpha), \quad u\left(\phi_{a}, \phi_{b}\right)=u_{a b} .
$$

Then $\pi u(\alpha, \beta) / h$ is the signed angle between the $s=1$ projections of $\alpha$ and $\beta$, and (3.6) becomes

$$
Z_{a b}^{(G)} \sim \sum_{\beta \in \Gamma_{b}^{+}}\left(\lambda_{a}, \beta\right) \chi_{1,1+u\left(\phi_{a}, \beta\right)},
$$

where $\Gamma_{b}^{+}$is the intersection of $\Gamma_{b}$, the $w$-orbit of $\phi_{b}$, with $\Phi^{+}$, the set of positive roots. That $u(\alpha, \beta)$ has only been defined modulo $2 h$ causes no ambiguities since $\chi_{r, s+2 h}=\chi_{r, s}$ (recall that the characters involved here are those for Virasoro central charge $1-6 / h(h-1)$ ).

Suitably reinterpreted, the expressions (3.6) and (3.9) apply equally to the expansions of partition functions in finite geometries, and to the decompositions of the modified traces, equation (1.4). For these two applications, the modulo $2 h$ ambiguity in $u(\alpha, \beta)$ should be removed by imposing $0 \leq u(\alpha, \beta)<2 h$, since $\lambda=1+u\left(\phi_{a}, \beta\right)$ really should only run from 1 to $h-1$.

To close this section, a remark on a curious coincidence. In refs. [16, 17] general formulae were given for the S-matrix elements of the (simply-laced) affine Toda field theories, 
and of certain perturbed conformal field theories. In either category, there is a theory associated with each simply-laced Lie algebra $G$, having $r=\operatorname{rank}(G)$ particle types, one for each node on the Dynkin diagram of $G$. The scattering amplitude for a pair of particles, of types $a$ and $b$, is given by

$$
S_{a b}(\theta)=\prod_{\beta \in \Gamma_{b}^{+}}\left\{1+u\left(\phi_{a}, \beta\right)\right\}^{\left(\lambda_{a}, \beta\right)},
$$

a product of a number of functions $\{\}.(\theta)$ of the rapidity $\theta$. The precise forms of these building blocks differ between the affine Toda and perturbed conformal cases; they can be found in [23]. (In fact, the formulae in [16,17 involved products taken over the entire orbit $\Gamma_{b}$ of certain sub-blocks $\{.\}_{ \pm}$, but it is easy to see that they are equivalent to (3.10).) The formal similarity between (3.10) and (3.9) should be clear, and gives a small practical application of the observation (3.1): should the numerical values of the coefficients $V_{a b}^{\lambda}$ ever happen to be needed, they can be read from the complete tables of the affine Toda S-matrix elements given in [23]. Whether this similarity is any more than a coincidence remains to be seen.

\section{Connections with the McKay correspondence}

The McKay correspondence [24] (see also [25,26]) is a bijection between the finite subgroups of $S U(2)$ and the affine Dynkin diagrams of types $\widehat{A}, \widehat{D}$ and $\widehat{E}$, such that to each of the finitely-many irreducible representations $\gamma_{a}$ of a given finite subgroup $\Gamma$ there is associated a node $a$ on the corresponding affine Dynkin diagram $\widehat{G}$. This association is encoded as follows: if $\gamma$ is the two-dimensional representation of $\Gamma$ provided by the original $S U(2)$, then

$$
\gamma \otimes \gamma_{a}=\sum I_{a b}^{(\widehat{G})} \gamma_{b}
$$

where $I^{(\widehat{G})}$ is the incidence matrix of $\widehat{G}\left(\right.$ so $\left.C^{(\widehat{G})}=2-I^{(\widehat{G})}\right)$. The trivial representation $\gamma_{0}$ is always associated with the 'extra' spot of $\widehat{G}$, corresponding to the negative of the highest root for the non-affine algebra $G$.

The irreducible representations of $S U(2)$ itself are infinite in number, there being one $\left(\pi_{n}\right.$ say) for every $n=0,1,2, \ldots$, of dimension $n+1$. On restriction these provide representations $\pi_{n} \mid \Gamma$ of the finite subgroups $\Gamma$, which may now be reducible. In [26], Kostant studied the decompositions

$$
\pi_{n} \mid \Gamma=\sum_{b} m_{n}^{b} \gamma_{b}
$$


of these representations into $\Gamma$-irreducibles. In particular, for each irreducible representation $\gamma_{b}$ of $\Gamma$ he computed the Poincaré series $P_{\Gamma}(t)_{b}$, encoding the multiplicities $m_{n}^{b}$ as

$$
P_{\Gamma}(t)_{b}=\sum_{n=0}^{\infty} m_{n}^{b} t^{n}
$$

and found

$$
P_{\Gamma}(t)_{b}=\frac{z(t)_{b}}{\left(1-t^{A}\right)\left(1-t^{B}\right)},
$$

where $A$ and $B$ are two $\Gamma$-dependent integers constrained by $A+B=h+2, A B=2|\Gamma|$, and $z(t)_{b}$ is a polynomial in $t$, of degree at most $h$. For the trivial representation $\gamma_{0}, z(t)_{0}=$ $1+t^{h}$, while for the remaining representations $\gamma_{b}$, for which the index $b$ also identifies a spot on the non-affine diagram, the expression for $z(t)_{b}$ invokes root system ideas very close to those described in section 2. To give the explicit formula, a little extra notation is needed. First, the two-colouring $\Delta=\left\{\alpha_{\bullet}\right\} \cup\left\{\alpha_{\circ}\right\}$ is alternatively labelled as $\Delta=\Delta_{1} \cup \Delta_{2}$, with the requirement that all the simple roots in $\Delta_{2}$ should be orthogonal to $\psi$, the highest root of $G$. As is clear from the form of the affine diagrams, this can be arranged for all root systems except $A_{\text {even }}$, a case which Kostant explicitly excluded. Correspondingly, $w_{\{\bullet\}}$ and $w_{\{\circ\}}$ are rewritten as $w_{1}$ and $w_{2}$ (not necessarily respectively), the requirement on $\Delta_{2}$ implying that $w_{2} \psi=\psi$. For $n \in \mathbf{Z}_{+}$, let $w_{n}=w_{1}$ if $n$ is odd and $w_{n}=w_{2}$ if $n$ is even $\left(w_{n}\right.$ should not be confused with the simple Weyl reflections, denoted $r_{a}$ above), and finally set $w^{[n]}=w_{n} w_{n-1} \ldots w_{1}, w^{[-n]}=w_{1} w_{2} \ldots w_{n}=\left(w^{[n]}\right)^{-1}$. Then for $b \neq 0$,

$$
z(t)_{b}=\sum_{n=1}^{h-1}\left(\lambda_{b}, w^{[n-1]} \psi-w^{[n]} \psi\right) t^{n}
$$

(Kostant also gave various other forms for this expression, but (4.5) is the most relevant here.)

Since the expression (4.5) uses machinery similar to that employed in earlier sections, the observation of Di Francesco and Zuber [27] that the coefficients of the $z(t)_{b}$ were to be found as certain of the $V_{a b}^{\lambda}$ should not now be too surprising. The remainder of this section will show precisely how this works, drawing on various ideas from [26] to put (4.5) into a form closer to (3.1).

To start, rewrite the coefficient of $t^{n}$ in (4.5) as

$$
z_{b, n}=\left(\psi, w^{[-(n-1)]}\left(\lambda_{b}-w_{n} \lambda_{b}\right)\right)
$$


Now $w_{n} \lambda_{b}$ is equal to $\lambda_{b}-\alpha_{b}$ or $\lambda_{b}$, depending on whether the $b$ is associated with the same subset $\left(\Delta_{1}\right.$ or $\left.\Delta_{2}\right)$ as $w_{n}$ or not. Thus roughly half of the coefficients $z_{b, n}$ are zero. To avoid overburdening the notation, assume for the time being that $\left\{\alpha_{\bullet}\right\}=\Delta_{2},\left\{\alpha_{\circ}\right\}=\Delta_{1}$ (this may conflict with (2.2), but recall that that particular choice only affected intermediate stages of the working and not the final results). Thus $\alpha_{b} \in \Delta_{2-u_{b}}$, and $\lambda_{b}-w_{n} \lambda_{b}$ is nonzero only if $n$ modulo 2 is equal to $2-u_{b}$, that is if $n=2 p+2-u_{b}$ for some $p$; in such cases it is equal to $\alpha_{b}$. Referring back to (2.13), it is straightforwardly checked that, whatever the colour of $\alpha_{b}$,

$$
w^{\left[-\left(2 p+1-u_{b}\right)\right]} \alpha_{b}=w^{-p} \phi_{b}
$$

and so equation (4.5) becomes

$$
z(t)_{b}=\sum_{1 \leq 2 p+2-u_{b} \leq h-1}\left(\psi, w^{-p} \alpha_{b}\right) t^{2 p+2-u_{b}} .
$$

Now for the $\widehat{D}$ and $\widehat{E}$ affine diagrams, the negative of the highest root joins to the remaining, non-affine part of the diagram by just a single link, connecting it to the simple root $\alpha_{f}$ say. Hence $\left(\psi, \alpha_{a}\right)=\delta_{a f}$, and so $\psi=\lambda_{f}$. For $\widehat{A}_{\text {odd }}$, the remaining case, $\psi$ has inner product 1 with both extremal roots on the non-affine diagram ( $\alpha_{f}$ and $\alpha_{\bar{f}}$ say), and so is equal to $\lambda_{f}+\lambda_{\bar{f}}$. In either case, the specification of $\Delta_{2}$ means that $\alpha_{f}$ and/or $\alpha_{\bar{f}}$ belong to $\Delta_{1}=\left\{\alpha_{\circ}\right\}$, so $u_{f}=u_{\bar{f}}=1$ and $2 p+2-u_{b}$ can be replaced by $2 p+1+u_{f b}$ or $2 p+1+u_{\bar{f} b}$. Substituting all of this into (4.8), comparing with (3.1) and recalling that the $V^{\lambda}$ 's not accounted for by (3.1) are automatically zero establishes that

$$
z_{b, n}=V_{f b}^{n}
$$

for $D$ and $E$, while

$$
z_{b, n}=V_{f b}^{n}+V_{\bar{f} b}^{n}
$$

for $A_{\text {odd }}$. Equations (4.9) and (4.10) exactly reproduce the observations of [27]. (Interestingly, (4.10) also holds for $A_{\text {even }}$.) The choice to set $\left\{\alpha_{\bullet}\right\}=\Delta_{2},\left\{\alpha_{\circ}\right\}=\Delta_{1}$ clearly should have no bearing on these final results, and indeed it is not too hard to check explicitly that (3.1) is unchanged if the black and white roots are swapped - the only points to note are that such a swap negates $u_{a b}$, sends $w$ to its inverse and changes the definition (2.13) of each $\phi_{a}$.

Given the correspondence between (3.10) and (3.9), the considerations of this section also apply to the exact S-matrices of affine Toda type; this was (very briefly) mentioned as a 'note added' in ref. [17]. 


\section{Conclusions}

These conclusions fall naturally into two parts: first, questions that remain in the $\widehat{S U(2)}$ case; and second, the (potentially more interesting) question of generalising the above constructions to other models associated with Lie algebras of higher rank.

To start the discussion for $\widehat{S U(2)}$, it is worth recalling that the Pasquier models in their continuum limits provide representatives for many of the unitary $c<1$ conformal field theories classified by Cappelli, Itzykson and Zuber [28], but not all of them. For central charge $c=1-1 / h(h-1)$, the possible modular invariant partition functions are labelled by a pairs $\left(G, G^{\prime}\right)$ of Lie algebras, with Coxeter numbers $h-1$ and $h$ respectively this forces one of $G, G^{\prime}$ to be of type $A$. The torus partition function of the Pasquier model associated with the algebra $G$ (of Coxeter number $h$ ) corresponds to the pair $\left(A_{h-2}, G\right.$ ) in the continuum limit, and so theories labelled by the pair $\left(G_{h-1}, A_{h-1}\right)$ are missed. However, lattice models have now been found which are expected to yield the $(G, A)$ partition functions [29]. It would be interesting to generalise the calculations of [5] to cover these models, and to find out whether the expansions corresponding to (1.2) also hide geometrical features similar to those outlined above.

Partition functions on the torus, and the associated issues of modular invariance [30], may seem rather disconnected from the discussions above of partition functions on surfaces with boundaries. There are at least two reasons why this is not so. As already mentioned in the introduction, Cardy [2] has established that in certain cases the link between boundary conditions, fusion rules and modular invariance is rather close. Unfortunately, this requires knowledge of expansions analogous to (1.2) for a complete set of boundary states invariant under the maximally-extended chiral algebra of the theory, which is generally larger than just Virasoro. Furthermore, the expansions should be in characters of this larger algebra. These conditions are not met by the expansions (1.2) beyond the (rather trivial) $A$ case, so it is not possible to apply Cardy's arguments directly here. Of more immediate relevance is the second point, an empirical observation made in [9] that certain of the $V^{\lambda}$ 's encode the decomposition of the extended conformal blocks for many modular invariant partition functions, thereby probing a finer structure than that revealed by examining characters of the maximal algebra alone. To be a little more precise, attention should first be restricted to the so-called 'type I' theories [6], that is theories for which the toroidal partition function is diagonal, a sum of squared moduli (the same restriction applied to the discussion in [2], in fact). For the $(A, G)$ or $(G, A) c<1$ theories, or for the $\widehat{S U(2)})_{k}$ affine Kac-Moody models 
labelled by a single algebra $G_{k}$, type I partition functions are found for $G=A, D_{\text {even }}, E_{6}$ and $E_{8}$. In these cases, a particular subset $T$ of the nodes of the Dynkin diagram of $G$ is chosen (these subsets are listed in [11]), and in addition a special node $a_{0}$ is picked, such that $q_{a_{0}}^{(1)}$ is minimal (alternatively put, $a_{0}$ labels the lightest particle in the corresponding affine Toda theory). Then the partition function on the torus is simply

$$
Z=\sum_{b \in T} Z_{b}
$$

where (in obvious notation)

$$
Z_{b}^{(A, G)}=\frac{1}{2} \sum_{r=1}^{h-2}\left|\sum_{\lambda=1}^{h-1} V_{a_{0} b}^{\lambda} \chi_{r, \lambda}\right|^{2} ; \quad Z_{b}^{(G, A)}=\frac{1}{2} \sum_{s=1}^{h-1}\left|\sum_{\lambda=1}^{h-2} V_{a_{0} b}^{\lambda} \chi_{\lambda, s}\right|^{2}
$$

for the $c<1$ models, while

$$
Z_{b}^{(G)}=\left|\sum_{\lambda=1}^{h-1} V_{a_{0} b}^{\lambda} \chi_{\lambda}\right|^{2}
$$

for an affine $\widehat{S U(2)}$ modular invariant, with $\chi_{\lambda}$ an affine rather than Virasoro character in this case. Combining these expressions with the formula (3.1) for the $V^{\lambda}$ 's shows that the geometry of root systems certainly has a rôle to play in the construction of the $A D E$ modular invariants, though there are clearly many elements of this which are obscure. In particular, it would be interesting to find a geometrical interpretation of the type I / type II distinction, and for the special subset $T$ of simple roots referred to above. An understanding of the modifications necessary to Cardy's arguments to cope with the expansions (1.2) might be a help in this regard, as might a direct derivation of the Virasoro decomposition of the Pasquier model toroidal partition functions from the lattice models, analogous to that achieved by Saleur and Bauer [5] on the cylinder as it stands the observation does shed a little light on some of the $A D E$ numerology that has been observed among the modular invariants. For example, in [32] it was noted that the conformal blocks for the $E_{8}$ modular invariants can be read from two of the Poincaré polynomials of the binary icosahedral subgroup of $S U(2)$. In the case of $E_{8}$, the node $a_{0}$ relevant for the modular invariants is the same as the node $f$ which arose in the discussion

3 Note, the treatment given by Pasquier in [31] is rather more indirect than this, in that he first relates the toroidal partition functions to sums of partition functions of certain other models, the so-called $f$-models. 
of the McKay correspondence in the previous section, and so the observation is consistent with equations (4.9) and (5.2). Note that this particular coincidence, between the Poincaré polynomials of a finite subgroup of $S U(2)$ and a modular invariant, does not generalise beyond $E_{8}$, consistent with the fact that it is only for this case that $a_{0}=f$.

The above have been rather specific questions. More generally, it would be good to have a better understanding of why there should be such a geometric interpretation for the quantities $V^{\lambda}$. For the purely elastic S-matrices, the general expression (3.10) seems less mysterious once it is seen how naturally such formulae solve the bootstrap equations. Given the formula (3.9) for the partition functions, an immediate thought is that there might be analogues of the bootstrap equations relating partition functions on the cylinder with different boundary conditions. However the variable $q$ seems a poor candidate to replace the rapidity $\theta$ in (3.10); much more natural would be to re-introduce the spectral parameter. This in turn is reminiscent of the close ties that exist between factorisable Smatrices and lattice models [33], ties which may ultimately explain the formal similarities between equations (3.10) and (3.9). Nevertheless, and despite various promising signs, a physically-motivated set of equations for the partition functions (1.2), or some small generalisation of them, has proved elusive. It may be that, just as the purely elastic Smatrices are too simple to exhibit any Yang-Baxter structure, so the bootstrap structure is absent for the Pasquier models, and would only be seen in some larger class of objects, within which both the purely elastic S-matrices and the Pasquier models would be found as degenerate special cases.

Finally to the question of generalisations beyond the $c<1$ Pasquier models, to intertwiners associated with algebras of higher rank than $S U(2)$. The approach adopted in [6,7] was to search for algebraic and graph-theoretic features of the Pasquier models and their intertwiners, and then to place these in a wider context. More general graphs than the simply-laced Dynkin diagrams arose, but nevertheless formulae exactly analogous to (1.3) were found, for which many of the features described above (in particular nonnegativity) continued to hold. The main point of this paper has been that, to understand the intertwiners in the $\widehat{S U(2)}$ case, it is necessary to add some geometrical insight to the algebra and graph theory. It would be very interesting if geometrical structures could be found lying behind the many mysterious results found in [6,7] for the higher-rank algebras, and indeed to see what these structures might be. Since the case of $\widehat{S U(2)}$ has already exhausted all finite reflection groups, the search will have to be quite wide, and might perhaps lead to something genuinely new. 


\section{Acknowledgements}

I would like to thank M. Bauer, P. Di Francesco, C. Itzykson, V. Pasquier, N. Sochen and J.-B. Zuber for many helpful discussions and explanations of their work. I am grateful to the European Community for a grant under the EC Science Programme.

\section{References}

[1] J. L. Cardy, Nucl. Phys. B275 (1986) 200.

[2] J. L. Cardy, Nucl. Phys. B324 (1989) 581.

[3] E. Verlinde, Nucl. Phys. B300 (1988) 360.

[4] V.Pasquier, Nucl. Phys. B285 (1987) 162.

[5] H. Saleur and M. Bauer, Nucl. Phys. B320 (1989) 591.

[6] P. Di Francesco and J.-B.Zuber, Nucl. Phys. B338 (1990) 602.

[7] N. Sochen, Nucl. Phys. B360 (1991) 613.

[8] V.Pasquier and H. Saleur, Nucl. Phys. B330 (1990) 523.

[9] P. Di Francesco and J.-B. Zuber, 'SU(N) lattice integrable models and modular invariance', Proceedings of the Trieste conference on recent developments in conformal field theories, October 1989.

[10] H. Saleur and J.-B. Zuber, 'Integrable Lattice Models and Quantum Groups', Proceedings of the 1990 Trieste Spring School on String Theory and Quantum Gravity.

[11] P. Di Francesco, Int. J. Mod. Phys. A7 (1992) 407.

[12] R.Steinberg, Trans. Amer. Math. Soc. 91 (1959) 493.

[13] B. Kostant, Am. J. Math. 81 (1959) 973.

[14] N. Bourbaki, Groupes et algèbres de Lie IV, V, VI, (Hermann, Paris 1968).

[15] R. Carter, Simple Groups of Lie Type, (Wiley 1972).

[16] P. E. Dorey, Nucl. Phys. B358 (1991) 654.

[17] P. E. Dorey, Nucl. Phys. B374 (1992) 741.

[18] E. Corrigan and P. E. Dorey, Phys. Lett. B273 (1991) 237.

[19] W. Lerche and N. P. Warner, Nucl. Phys. B358 (1991) 571.

[20] M. D. Freeman, Phys. Lett. B261 (1991) 57;

A. Fring, H. C. Liao and D. I. Olive, Phys. Lett. B266 (1991) 82.

[21] H. W. Braden, J. Phys. A25 (1992) L15.

[22] A. Fring and D.I. Olive, 'The fusing rule and the scattering matrix of affine Toda theory', preprint Imperial/TP-91-92/08.

[23] H. W. Braden, E. Corrigan, P. E. Dorey and R. Sasaki, Nucl. Phys. B338 (1990) 689.

[24] J. McKay, Proc. Symp. Pure Math. 37 (1980) 183.

[25] R.Steinberg, Pacific J. Math. 118 (1985) 587.

[26] B. Kostant, Proc. Natl. Acad.Sci. USA 81 (1984) 5275;

B. Kostant, Astérisque, hors série, 1985, 209 - 255. 
[27] P. Di Francesco and J.-B. Zuber, unpublished, reported in [11].

[28] A. Cappelli, C. Itzykson and J-B Zuber, Comm. Math. Phys. 113 (1987) 1.

[29] P. Roche, 'On the construction of integrable dilute A-D-E models', 1992 ENSLAPP preprint.

[30] J. L. Cardy, Nucl. Phys. B270 (1986) 186.

[31] V.Pasquier, J. Phys. A20 (1987) L1229.

[32] C. Itzykson, Advanced Studies in Pure Mathematics 19 (1989) 287.

[33] A. B. Zamolodchikov, Sov. Sci. Rev., Physics, v.2 (1980). 
Figure 1

A general eigenspace of the Coxeter element, showing the actions of $w_{\{\bullet\}}, w_{\{0\}}$ and $w$.

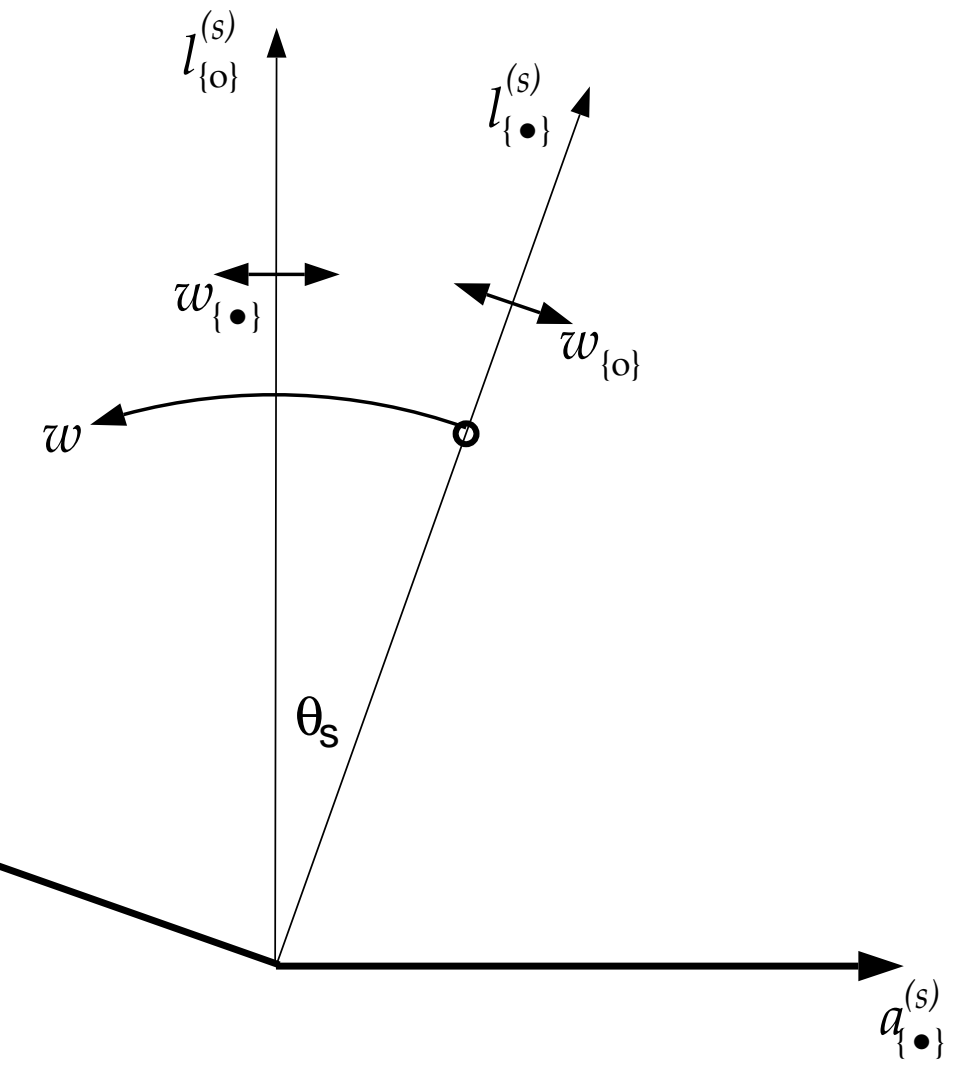


Figure 2

The $s=1$ eigenspace of the Coxeter element, showing the regions into which the positive and negative roots project.

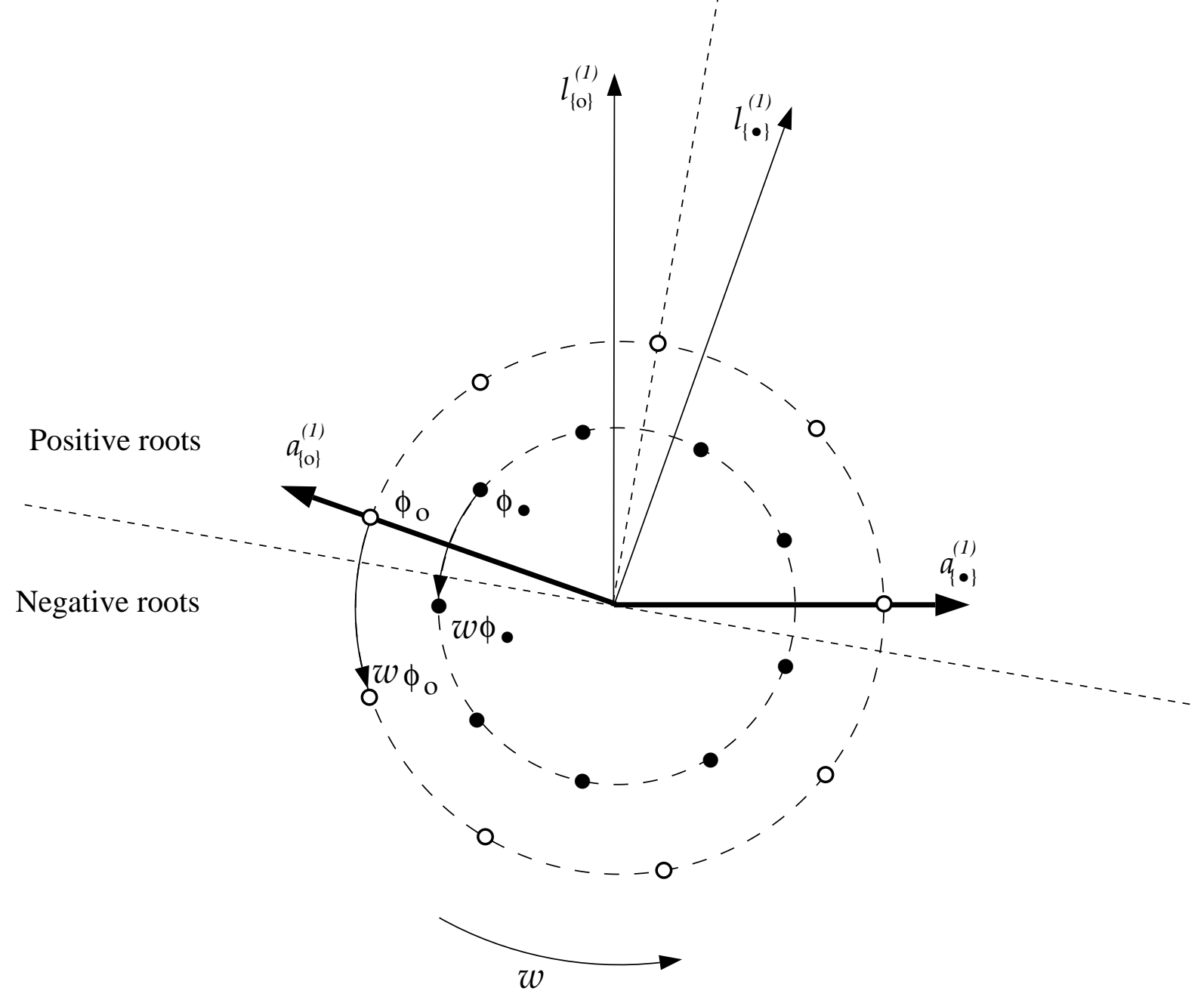

